\title{
GEOMETRY OF ISOPARAMETRIC HYPERSURFACES IN RIEMANNIAN MANIFOLDS*
}

\author{
JIANQUAN GE ${ }^{\dagger}$ AND ZIZHOU TANG ${ }^{\dagger}$
}

\begin{abstract}
In our previous work, we studied isoparametric functions on Riemannian manifolds, especially on exotic spheres. One result there says that, in the family of isoparametric hypersurfaces of a closed Riemannian manifold, there exists at least one minimal isoparametric hypersurface. In this paper, we show such a minimal isoparametric hypersurface is also unique in the family if the ambient manifold has positive Ricci curvature. Moreover, we give a proof of Theorem D claimed by Q.M.Wang (without proof) which asserts that the focal submanifolds of an isoparametric function on a complete Riemannian manifold are minimal. Further, we study isoparametric hypersurfaces with constant principal curvatures in general Riemannian manifolds. It turns out that in this case the focal submanifolds have the same properties as those in the standard sphere, i.e., the shape operator with respect to any normal direction has common constant principal curvatures. Some necessary conditions involving Ricci curvature and scalar curvature are also derived.
\end{abstract}

Key words. Isoparametric function, focal submanifold, constant mean curvature.

AMS subject classifications. $53 \mathrm{C} 20$.

1. Introduction. A non-constant smooth function $f: N \rightarrow \mathbb{R}$ defined on a smooth connected Riemannian manifold $N$ is called transnormal if there is a smooth function $b: J \rightarrow \mathbb{R}$ such that

$$
|\nabla f|^{2}=b(f)
$$

where $J=f(M) \subset \mathbb{R}$ and $\nabla f$ is the gradient of $f$. If moreover there is a continuous function $a: J \rightarrow \mathbb{R}$ such that

$$
\triangle f=a(f)
$$

where $\triangle f$ is the Laplacian of $f$, then $f$ is called isoparametric (cf. [Wa87], [GT09]). Equation (1) means that the regular hypersurfaces $M_{t}:=f^{-1}(t)$ (where $t$ is a regular value of $f$ ) are parallel and (2) says that these parallel hypersurfaces have constant mean curvatures. These regular level hypersurfaces $M_{t}:=f^{-1}(t)$ of an isoparametric function $f$ are called isoparametric hypersurfaces. A transnormal function $f$ on a complete Riemannian manifold has no critical value in $\operatorname{Int}(J)$ ([Wa87]). The preimage of the maximum (resp. minimum), if it exists, of an isoparametric (or transnormal) function $f$ is called the focal variety of $f$, denoted by $M_{+}\left(\right.$resp. $\left.M_{-}\right){ }^{1}$

A fundamental structural result given by [Wa87] says that the focal varieties $M_{ \pm}$ of a transnormal function on a complete Riemannian manifold are smooth submanifolds and each regular level hypersurface $M_{t}$ is a tubular hypersurface over either of

${ }^{*}$ Received January 8, 2012; accepted for publication September 28, 2012. The project is partially supported by the NSFC (No. 11071018 and No. 11001016), the SRFDP (No. 20100003120003), and the Program for Changjiang Scholars and Innovative Research Team in University.

†School of Mathematical Sciences, Laboratory of Mathematics and Complex Systems, Beijing Normal University, Beijing 100875, China (jqge@bnu.edu.cn; zztang@mx.cei.gov.cn).

${ }^{1}$ In our papers, for simplicity we always assume in the definition that $b$ is smooth, i.e., $C^{\infty}$. In fact, all the results go through when $b$ is merely $C^{2}$ as remarked in [Wa87]. In private discussions, G. Thorbergsson pointed out that it should also work for $b$ being a continuous function in the case of both equations (1-2) are satisfied, since then there should exist some other function $\tilde{f}$ with the same level sets as $f$ such that it satisfies equations (1-2) for some smooth $\left(C^{2}\right)$ function $b$. 
$M_{ \pm}$. In our previous work [GT09], we called an isoparametric (or transnormal) function proper if each component of $M_{ \pm}$has codimension not less than 2. For a properly isoparametric function, $M_{ \pm}$are just the focal sets of each regular level hypersurface $M_{t}$ and all level sets are connected. Moreover, there we proved that at least one properly isoparametric hypersurface is minimal if the ambient space $N$ is closed. In this paper, by using the Riccati equation, we observe that such a minimal isoparametric hypersurface is also unique in its family if $N$ has positive Ricci curvature. Next, we express the shape operator $S(t)$ of $M_{t}$ as a power series with respect to the distance between $M_{t}$ and $M_{ \pm}$. As an immediate consequence, we can give a complete proof to Theorem D of [Wa87] (without proof there):

THEOREM 1.1. ${ }^{2}$ The focal varieties $M_{ \pm}$of an isoparametric function $f$ on a complete Riemannian manifold $N$ are minimal submanifolds.

In fact, by combining Wang's structural result [Wa87], one can see that under an additional assumption that $N$ and $M_{ \pm}$are all compact, Mazzeo and Pacard [MP05] have essentially proved the above theorem as a special case in their Theorem 6.1 by an argument from geometric measure theory which is not fit for the noncompact case. In private communications, we learned that Miyaoka (cf. [Mi12]) is also concerned with a complete proof of Theorem 1.1. Further study of the power series expression establishes

THEOREM 1.2. Suppose that each isoparametric hypersurface $M_{t}$ has constant principal curvatures ${ }^{3}$ with respect to the unit normal vector field in the direction of $\nabla f$. Then each of the focal varieties $M_{ \pm}$has common constant principal curvatures in all normal directions, i.e., the eigenvalues of the shape operator are constant and independent of the choices of the point and unit normal vector of $M_{ \pm}$.

As a corollary, the principal curvatures of such focal varieties occur as pairs of opposite signs and thus $M_{ \pm}$are austere (minimal) submanifolds in the sense of [HL82]. As byproducts, we obtain some necessary curvature conditions for a manifold to admit (certain) isoparametric functions in Corollary 3.1 and Corollary 3.2 in Section 3.

Submanifolds with constant principal curvatures are regarded as the analogue in submanifold geometry to locally symmetric spaces in Riemannian geometry in [BCO03] where the normal holonomy theory takes important role in the studies of such submanifolds in space forms. It is worth mentioning that the normal holonomy theory for submanifolds in general Riemannian manifolds has not been fully developed though it seems fruitful in many areas (cf. [Br99], [CDO08], etc.). Note that Theorem 1.1 and Theorem 1.2 generalize corresponding results in the classical theory of isoparametric hypersurfaces in real space forms (cf. [No73], [Mü80]), recalling that, a hypersurface $M^{n}$ in a real space form $N^{n+1}(c)$ with constant sectional curvature $c$ is said to be isoparametric if it has constant principal curvatures. Cartan ([Car38],[Car39]) and Münzner ([Mü80]) showed that such an isoparametric hypersurface belongs to a family of parallel hypersurfaces of constant mean curvature which consists of regular level sets of a homogeneous polynomial (called isoparametric polynomial) satisfying the socalled Cartan-Münzner equation. Provided with the "good symmetry" of space forms, Münzner [Mü80] could obtain more specific properties about principal curvatures of

\footnotetext{
${ }^{2}$ Note added in proof: Very recently we happened to see an unpublished paper by Ni [Ni97] from internet which gave another different proof of Theorem 1.1.

${ }^{3}$ When $M_{t}$ is disconnected, we assume that all connected components have common constant principal curvatures, or equivalently, the principal curvatures of $M_{t}$ depend only on $\left.f\right|_{M_{t}}=t$.
} 
the focal varieties and isoparametric hypersurfaces than Theorem 1.2 does, on which they were heavily depended in the proof of his splendid result about the number $g$ of distinct principal curvatures of isoparametric hypersurfaces in spheres (For the theory of isoparametric hypersurfaces in the model space forms, see [Th00] for an excellent survey and see [CCJ07], [Imm08], [GX10] for recent progresses and applications).

2. The shape operator of Tubes. In this section we use similar definitions and notations as in [Gr04] and [MMP06]. Let $P^{m} \subset M^{n}$ be an $m$-dimensional embedded submanifold of an $n$-dimensional Riemannian manifold $M$. For a given $p \in P$, we now introduce Fermi coordinates in a neighborhood $\mathcal{U}^{\prime}$ of $p$ in $M$. First we choose normal geodesic coordinates $\left(y_{1}, \cdots, y_{m}\right)$ centered at $p$ in a neighborhood $\mathcal{U}$ of $p$ in $P$. Then in $\mathcal{U}$ we fix an orthonormal sections $E_{m+1}, \cdots, E_{n}$ of the normal bundle $\mathcal{V}$ of $P$ in $M$ such that they are parallel with respect to the normal connection along any geodesic ray from $p$ in $P$. The Fermi coordinates $\left(x_{1}, \cdots, x_{n}\right)$ of $(\mathcal{U} \subset P \subset) \mathcal{U}^{\prime} \subset M$ centered at $p$ are defined by

$$
\begin{aligned}
& x_{a}\left(\exp _{p^{\prime}}\left(\sum_{j=m+1}^{n} t_{j} E_{j}\left(p^{\prime}\right)\right)\right)=y_{a}\left(p^{\prime}\right) \quad(a=1, \cdots, m), \\
& x_{i}\left(\exp _{p^{\prime}}\left(\sum_{j=m+1}^{n} t_{j} E_{j}\left(p^{\prime}\right)\right)\right)=t_{i} \quad(i=m+1, \cdots, n),
\end{aligned}
$$

for $p^{\prime} \in \mathcal{U}$ and any sufficiently small numbers $t_{m+1}, \cdots, t_{n}$. From the definitions, it is easily seen that the coordinates vector fields $\partial x_{1}, \cdots, \partial x_{n}$ satisfy

$$
\begin{aligned}
\left.\nabla_{\partial x_{a}} \partial x_{b}\right|_{p} \in \mathcal{V}_{p} P, & \left.\nabla_{\partial x_{a}} \partial x_{i}\right|_{p} & \in \mathcal{T}_{p} P, & \left.\nabla_{\partial x_{i}} \partial x_{j}\right|_{\mathcal{U}}=0, \\
\left.\left\langle\partial x_{\alpha}, \partial x_{\beta}\right\rangle\right|_{p}=\delta_{\alpha \beta}, & \left.\left\langle\partial x_{a}, \partial x_{i}\right\rangle\right|_{\mathcal{U}} & =0, & \left.\left\langle\partial x_{i}, \partial x_{j}\right\rangle\right|_{\mathcal{U}}=\delta_{i j},
\end{aligned}
$$

where $\nabla$ denotes the covariant derivative in $M,\langle\cdot, \cdot\rangle$ denotes the metric, and the indices convention is that indices $a, b, c, \cdots \in\{1, \cdots, m\}$, indices $i, j, k, \cdots \in\{m+$ $1, \cdots, n\}$ and indices $\alpha, \beta, \gamma, \cdots \in\{1, \cdots, n\}$.

In terms of Fermi coordinates, the distance function, say $\sigma$, to $P$ in $M$ and the (outward) unit normal vector field, say $N$, of any tubular hypersurface $P_{t}$ at a distance $\sigma=t>0$ from $P$ can be written as

$$
\sigma\left(q^{\prime}\right)=\sqrt{\sum_{i=m+1}^{n} x_{i}^{2}} \text { and } N\left(q^{\prime}\right)=\sum_{i=m+1}^{n} \frac{x_{i}}{\sigma} \frac{\partial}{\partial x_{i}} \triangleq \nabla \sigma
$$

for $q^{\prime}=\exp _{p^{\prime}}\left(\sum_{j=m+1}^{n} x_{j} E_{j}\left(p^{\prime}\right)\right) \in P_{t} \subset \mathcal{U}^{\prime}-P$, where the last equality $(\triangleq)$ is just the Generalized Gauss Lemma.

The shape operator $S(t)$ of $P_{t}$ with respect to $N$ at $q^{\prime} \in P_{t}$ is just the restriction to $P_{t}$ of the tensorial operator $S: \mathcal{T}\left(\mathcal{U}^{\prime}-P\right) \rightarrow \mathcal{T}\left(\mathcal{U}^{\prime}-P\right)$ defined by

$$
S U=-\nabla_{U} N
$$

for $U \in \mathcal{T}_{q^{\prime}}\left(\mathcal{U}^{\prime}-P\right)$. It is easily seen that $S$ is symmetric and $S N=0$. Then covariant derivative of $S(t)$ along normal geodesic of $P_{t}$ gives the Riccati equation:

$$
S^{\prime}(t)=S(t)^{2}+R(t),
$$


where $R(t) U=R_{N U} N=\left(\nabla_{[N, U]}-\left[\nabla_{N}, \nabla_{U}\right]\right) N$ for $U \in \mathcal{T}_{q^{\prime}} P_{t}$. Taking trace shows that

$$
H^{\prime}(t)=\|S(t)\|^{2}+\rho^{M}(N, N),
$$

where $H(t)=\operatorname{Trace}(S(t))$ is the mean curvature of $P_{t}, \rho^{M}$ is the Ricci curvature of $M$ and $\|\cdot\|$ is the norm induced from the metric. Therefore, if the Ricci curvature $\rho^{M}$ is positive, $H(t)$ is a strictly increasing function as $t$ grows. Recall that in [GT09], we showed that at least one is minimal among a family of properly isoparametric hypersurfaces in a closed Riemannian manifold. Combining these with the structural result of [Wa87], we get

COROLLARY 2.1. There exists a unique minimal isoparametric hypersurface among a family of properly isoparametric hypersurfaces in a closed Riemannian manifold of positive Ricci curvature.

REMARK 2.1. In Proposition 4.1 of [GT09], we found isoparametric functions which are proper on each Milnor sphere, and Remark 4.2 of [GT09] told that in that situation the Milnor spheres could carry metrics of positive Ricci curvature (even nonnegative sectional curvature). So the above corollary tells that there is one and only one minimal isoparametric hypersurface in each family.

Now we come to derive the power series expansion formula for $S(t)$ with respect to $t$. For any normal vector $v \in \mathcal{V}_{p} P$, the shape operator $T_{v}$ of $P$ in direction $v$ at $p$ is defined by

$$
\left\langle T_{v}(X), Y\right\rangle=\left\langle\nabla_{X} Y, v\right\rangle=-\left.\left\langle\nabla_{X} V, Y\right\rangle\right|_{p}
$$

for any vector fields $X, Y$ tangent to $P$, where $V$ is any normal vector field with $\left.V\right|_{p}=v$. Let $\left\{T_{a b}^{v}\right\}$ denote the coefficients of $T_{v}$ under the coordinate vector fields, i.e.,

$$
T_{v}\left(\left.\partial x_{a}\right|_{p}\right)=\left.\sum_{b=1}^{m} T_{a b}^{v} \partial x_{b}\right|_{p}, \quad \text { or } \quad T_{a b}^{v}=\left.\left\langle T_{v}\left(\partial x_{a}\right), \partial x_{b}\right\rangle\right|_{p}
$$

Then we will also write $T_{v}=\left(T_{a b}^{v}\right)$ (as a matrix), and $T_{i}=T_{\left.\partial x_{i}\right|_{p}}=\left(T_{a b}^{i}\right)$ for simplicity. For any unit normal vector $v=\left.\sum_{j=m+1}^{n} v_{j} \partial x_{j}\right|_{p} \in \mathcal{V}_{p} P$, s.t., $\sum_{j=m+1}^{n} v_{j}^{2}=$ 1 , we denote by $\eta_{v}(t)=\exp _{p}(t v)$ the unique geodesic in direction $v$ through $p$ in $M$. Obviously, the Fermi coordinates of $\eta_{v}(t) \in P_{t}$ are $\left(0, \cdots, 0, t v_{m+1}, \cdots, t v_{n}\right)$, and

$$
\left.N\right|_{\eta_{v}(t)}=\eta_{v}^{\prime}(t)=\left.\sum_{j=m+1}^{n} v_{j} \partial x_{j}\right|_{\eta_{v}(t)}
$$

Let $g_{\alpha \beta}=\left\langle\partial x_{\alpha}, \partial x_{\beta}\right\rangle$ be the metric coefficients. Then we recall their power series expansion in preparation for that of the shape operator.

Lemma 2.1. At the point $\eta_{v}(t)=\exp _{p}(t v) \in P_{t}$, the following expansions hold

$$
\begin{aligned}
& g_{a b}(t)=\delta_{a b}-2 T_{a b}^{v} t+\left(-\left\langle R_{v \partial x_{a}} v, \partial x_{b}\right\rangle+\sum_{c} T_{a c}^{v} T_{c b}^{v}\right) t^{2}+O\left(t^{3}\right), \\
& g_{a i}(t)=-\frac{2}{3}\left\langle R_{v \partial x_{a}} v, \partial x_{i}\right\rangle t^{2}+O\left(t^{3}\right) \\
& g_{i j}(t)=\delta_{i j}-\frac{1}{3}\left\langle R_{v \partial x_{i}} v, \partial x_{j}\right\rangle t^{2}+O\left(t^{3}\right) .
\end{aligned}
$$


Proof. Though these formulas can be derived by standard calculations and some of them have occurred in the literature (cf.[MMP06]), we briefly prove the second here for the reader's convenience.

By formulas (3-4), $g_{a i}(0)=0$,

$$
g_{a i}^{\prime}(0)=v\left(\left\langle\partial x_{a}, \partial x_{i}\right\rangle\right)=\left\langle\nabla_{v} \partial x_{a}, \partial x_{i}\right\rangle+\left\langle\partial x_{a}, \nabla_{v} \partial x_{i}\right\rangle=0,
$$

and

$$
\begin{aligned}
g_{a i}^{\prime \prime}(0) & =\left.\frac{d}{d t}\left(\left.\left\langle\nabla_{N} \partial x_{a}, \partial x_{i}\right\rangle\right|_{\eta_{v}(t)}+\left.\left\langle\partial x_{a}, \nabla_{N} \partial x_{i}\right\rangle\right|_{\eta_{v}(t)}\right)\right|_{t=0} \\
& =v\left(\left\langle\nabla_{\widetilde{N}} \partial x_{a}, \partial x_{i}\right\rangle+\left\langle\partial x_{a}, \nabla_{\widetilde{N}} \partial x_{i}\right\rangle\right) \quad\left(\text { where } \widetilde{N}=\sum_{j=m+1}^{n} v_{j} \partial x_{j}\right) \\
& =\left\langle\nabla_{v} \nabla_{\widetilde{N}} \partial x_{a}, \partial x_{i}\right\rangle+\left\langle\partial x_{a}, \nabla_{v} \nabla_{\widetilde{N}} \partial x_{i}\right\rangle \\
& =-\left\langle R_{v \partial x_{a}} v, \partial x_{i}\right\rangle-\frac{1}{3}\left\langle R_{v \partial x_{i}} v, \partial x_{a}\right\rangle \quad \text { (by Lemma 9.20 in [Gr04]) } \\
& =-\frac{4}{3}\left\langle R_{v \partial x_{a}} v, \partial x_{i}\right\rangle
\end{aligned}
$$

where Lemma 9.20 in [Gr04] shows (by properties of Fermi coordinates and polarization)

$$
\begin{gathered}
\left.\nabla_{\partial x_{i}} \nabla_{\partial x_{j}} \partial x_{a}\right|_{p}=-\left.R_{\partial x_{i} \partial x_{a}} \partial x_{j}\right|_{p} \\
\left.\nabla_{\partial x_{i}} \nabla_{\partial x_{j}} \partial x_{k}\right|_{p}=-\left.\frac{1}{3}\left(R_{\partial x_{i} \partial x_{j}} \partial x_{k}+R_{\partial x_{i} \partial x_{k}} \partial x_{j}\right)\right|_{p} .
\end{gathered}
$$

Therefore, by Taylor formula, we get the second expansion formula. The other two can also be derived similarly.

Lemma 2.2. At the point $\eta_{v}(t)=\exp _{p}(t v) \in P_{t}$, the following expansions hold

$$
\begin{aligned}
\nabla_{\partial x_{a}} \partial x_{j}= & \sum_{b}-T_{a b}^{j} \partial x_{b}-t \sum_{b}\left(\left\langle R_{v \partial x_{a}} \partial x_{j}, \partial x_{b}\right\rangle+\sum_{c} T_{a c}^{j} T_{c b}^{v}\right) \partial x_{b} \\
& -t \sum_{k}\left\langle R_{v \partial x_{a}} \partial x_{j}, \partial x_{k}\right\rangle \partial x_{k}+\sum_{\alpha} O\left(t^{2}\right)_{\alpha} \partial x_{\alpha} \\
\nabla_{\partial x_{l}} \partial x_{j}= & -\frac{t}{3} \sum_{b}\left(\left\langle R_{v \partial x_{l}} \partial x_{j}, \partial x_{b}\right\rangle+\left\langle R_{v \partial x_{j}} \partial x_{l}, \partial x_{b}\right\rangle\right) \partial x_{b} \\
& -\frac{t}{3} \sum_{k}\left(\left\langle R_{v \partial x_{l}} \partial x_{j}, \partial x_{k}\right\rangle+\left\langle R_{v \partial x_{j}} \partial x_{l}, \partial x_{k}\right\rangle\right) \partial x_{k}+\sum_{\alpha} O\left(t^{2}\right)_{\alpha} \partial x_{\alpha} .
\end{aligned}
$$

Proof. By definitions,

$$
\left.\left\langle\nabla_{\partial x_{a}} \partial x_{j}, \partial x_{b}\right\rangle\right|_{p}=-T_{a b}^{j},\left.\quad\left\langle\nabla_{\partial x_{a}} \partial x_{j}, \partial x_{k}\right\rangle\right|_{p}=0 .
$$

Similarly as in the proof of Lemma 2.1, we have

$$
\begin{aligned}
v\left\langle\nabla_{\partial x_{a}} \partial x_{j}, \partial x_{b}\right\rangle & =\left\langle\nabla_{v} \nabla_{\partial x_{a}} \partial x_{j}, \partial x_{b}\right\rangle+\left\langle\nabla_{\partial x_{a}} \partial x_{j}, \nabla_{v} \partial x_{b}\right\rangle \\
& =-\left\langle R_{v \partial x_{a}} \partial x_{j}, \partial x_{b}\right\rangle+\sum_{c} T_{a c}^{j} T_{c b}^{v}
\end{aligned}
$$




$$
\begin{aligned}
v\left\langle\nabla_{\partial x_{a}} \partial x_{j}, \partial x_{k}\right\rangle & =\left\langle\nabla_{v} \nabla_{\partial x_{a}} \partial x_{j}, \partial x_{k}\right\rangle+\left\langle\nabla_{\partial x_{a}} \partial x_{j}, \nabla_{v} \partial x_{k}\right\rangle \\
& =-\left\langle R_{v \partial x_{a}} \partial x_{j}, \partial x_{k}\right\rangle .
\end{aligned}
$$

Suppose that

$$
\left.\nabla_{\partial x_{\alpha}} \partial x_{j}\right|_{\eta_{v}(t)}=\sum_{\alpha} \xi_{\alpha}(t) \partial x_{\alpha}
$$

Then

$$
\left.\left\langle\nabla_{\partial x_{a}} \partial x_{j}, \partial x_{\beta}\right\rangle\right|_{\eta_{v}(t)}=\sum_{\alpha} \xi_{\alpha}(t) g_{\alpha \beta}(t),
$$

and thus by Lemma 2.1, we get

$$
\begin{aligned}
& \xi_{b}(t)=-T_{a b}^{j}-t\left(\left\langle R_{v \partial x_{a}} \partial x_{j}, \partial x_{b}\right\rangle+\sum_{c} T_{a c}^{j} T_{c b}^{v}\right)+O\left(t^{2}\right), \\
& \xi_{k}(t)=-t\left\langle R_{v \partial x_{a}} \partial x_{j}, \partial x_{k}\right\rangle+O\left(t^{2}\right),
\end{aligned}
$$

which completes the proof of the first formula of the lemma.

The second formula can also be derived similarly. $\square$

Now we are ready to give the power series expansion of the shape operator. From Lemma 2.2 , one can immediately deduce the following.

LEMmA 2.3. At the point $\eta_{v}(t)=\exp _{p}(t v) \in P_{t}$, the following expansions hold

$$
\begin{aligned}
\nabla_{\partial x_{a}} N= & \sum_{b}-T_{a b}^{v} \partial x_{b}-t \sum_{b}\left(\left\langle R_{v \partial x_{a}} v, \partial x_{b}\right\rangle+\sum_{c} T_{a c}^{v} T_{c b}^{v}\right) \partial x_{b} \\
& -t \sum_{k}\left\langle R_{v \partial x_{a}} v, \partial x_{k}\right\rangle \partial x_{k}+\sum_{\alpha} O\left(t^{2}\right)_{\alpha} \partial x_{\alpha}, \\
\nabla_{\partial x_{l}} N= & \frac{1}{t} \partial x_{l}-\frac{v_{l}}{t} N-\frac{t}{3}\left(\sum_{b}\left\langle R_{v \partial x_{l}} v, \partial x_{b}\right\rangle \partial x_{b}+\sum_{k}\left\langle R_{v \partial x_{l}} v, \partial x_{k}\right\rangle \partial x_{k}\right) \\
& +\sum_{\alpha} O\left(t^{2}\right)_{\alpha} \partial x_{\alpha} .
\end{aligned}
$$

Notice that along the geodesic $\eta_{v}(t)$ one can always choose a system of Fermi coordinates $\left(x_{1}, \cdots, x_{n}\right)$ such that it satisfies a further property (Lemma 2.5 in [Gr04]):

$$
\left.\partial x_{n}\right|_{\eta_{v}(t)}=\eta_{v}^{\prime}(t)=\left.N\right|_{\eta_{v}(t)} .
$$

Then we denote by $\left(S_{\alpha \beta}\right)$ the coefficients of the operator $S$ defined by (5) under such Fermi coordinates, i.e., $S\left(\partial x_{\alpha}\right)=\sum_{\beta=1}^{n} S_{\alpha \beta} \partial x_{\beta}$. Note that $\left.S\left(\partial x_{n}\right)\right|_{\eta_{v}(t)}=\left.S(N)\right|_{\eta_{v}(t)}=$ 0 and $\left.\partial x_{n}\right|_{\eta_{v}(0)}=\eta_{v}^{\prime}(0)=v\left(\right.$ which implies $\left.v_{m+1}=\cdots=v_{n-1}=0, v_{n}=1\right)$. Therefore, from Lemma 2.3, we obtain

Proposition 2.1. At the point $\eta_{v}(t)=\exp _{p}(t v) \in P_{t}$, the following expansion holds

$$
S=\left(S_{\alpha \beta}\right)=\left(\begin{array}{ccc}
T_{v}+t A+\mathcal{O}\left(t^{2}\right) & t B+\mathcal{O}\left(t^{2}\right) & \mathcal{O}\left(t^{2}\right) \\
t C+\mathcal{O}\left(t^{2}\right) & -\frac{1}{t} I+t D+\mathcal{O}\left(t^{2}\right) & \mathcal{O}\left(t^{2}\right) \\
0 & 0 & 0
\end{array}\right)
$$


where $A=\left(\left\langle R_{v \partial x_{a}} v, \partial x_{b}\right\rangle+\sum_{c} T_{a c}^{v} T_{c b}^{v}\right)$ is a matrix with indices $a, b \in\{1, \cdots, m\}$; $D=\left(\frac{1}{3}\left\langle R_{v \partial x_{l}} v, \partial x_{k}\right\rangle\right)$ is a matrix with indices $l, k \in\{m+1, \cdots, n-1\} ; B=$ $\left(\left\langle R_{v \partial x_{a}} v, \partial x_{k}\right\rangle\right) ; C=\left(\frac{1}{3}\left\langle R_{v \partial x_{l}} v, \partial x_{b}\right\rangle\right) ; \mathcal{O}\left(t^{2}\right)$ denotes matrices with elements of order not less than 2 .

Recall that $S(t)$ is the restriction to $P_{t}$ of the operator $S$ and as a unit normal vector of $P_{t},\left.\partial x_{n}\right|_{\eta_{v}(t)}=N$ is an eigenvector of $S$ with corresponding eigenvalue 0 . Thus the expansion (7) implies

COROLlary 2.2. The principal curvatures of $P_{t}$ at $\eta_{v}(t)$ are just the eigenvalues of the matrix (with notations as above)

$$
\bar{S}(t):=\left(\begin{array}{cc}
T_{v}+t A+\mathcal{O}\left(t^{2}\right) & t B+\mathcal{O}\left(t^{2}\right) \\
t C+\mathcal{O}\left(t^{2}\right) & -\frac{1}{t} I+t D+\mathcal{O}\left(t^{2}\right)
\end{array}\right)
$$

3. Proof of Theorems and curvature restrictions. In this section, we apply the expansion formula of the shape operator (Corollary 2.2) to prove the theorems stated in the introduction. As byproducts, there are some curvature restrictions necessary for a Riemannian manifold to admit (certain) isoparametric functions.

Proof of Theorem 1.1. By the structural result of [Wa87], each regular level hypersurface $M_{s}=f^{-1}(s)$ of a transnormal function $f$ is a tubular hypersurface of either of the focal varieties $M_{ \pm}$(if non-empty). Then we could apply the results of last section here with $P=M_{-}$(alternatively $M_{+}$) and its tubular hypersurface $P_{t}$ at distance $t\left(0<t<\operatorname{dist}\left(M_{-}, M_{+}\right)\right)$from $P$ is just some regular level hypersurface of $f$. Since now $f$ is an isoparametric function, $P_{t}$ has constant mean curvature $H(t)$, which implies, by Corollary 2.2,

(8) $\operatorname{Trace}(\bar{S}(t))=\operatorname{Trace}\left(T_{v}\right)-\frac{n-m-1}{t}+t(\operatorname{Trace}(A)+\operatorname{Trace}(D))+O\left(t^{2}\right)=H(t)$

is independent of the choices of point $p \in P$ and unit normal vector $v \in \mathcal{V}_{p} P$. Therefore, comparing the coefficients of $t^{\lambda}$ 's, we know that $\operatorname{Trace}\left(T_{v}\right)$ is a constant independent of the choices of unit normal vector $v \in \mathcal{V}_{p} P$ and thus must vanish, since by linearity, $\operatorname{Trace}\left(T_{v}\right)=\operatorname{Trace}\left(T_{-v}\right)=-\operatorname{Trace}\left(T_{v}\right)$. This completes the proof of Theorem 1.1.

Formula (8) in the proof above also implies

(9) $\quad \Gamma_{P}:=\operatorname{Trace}(A)+\operatorname{Trace}(D)=\frac{1}{3}\left(\rho^{N}(v, v)+2 \sum_{a=1}^{m} K^{N}\left(v, \partial x_{a}\right)+3\left\|T_{v}\right\|^{2}\right)$

is a constant independent of the choices of point $p \in P$ and unit normal vector $v \in \mathcal{V}_{p} P$, where $\rho^{N}(v, v), K^{N}\left(v, \partial x_{a}\right)$ are the Ricci curvature in direction $v$ and the sectional curvature of the plane spanned by $\left(v, \partial x_{a}\right)$ of $N$ respectively. Moreover, by taking the Trace of (9) with respect to $v$ and by Gauss equation,

$$
\frac{1}{3} \sum_{i j=m+1}^{n} K_{i j}^{N}+\sum_{a b=1}^{m} K_{a b}^{N}+\sum_{i a} K_{i a}^{N}-R^{P}=(n-m) \Gamma_{P}
$$


is a constant function on $P$, where $K_{\alpha \beta}^{N}:=K^{N}\left(\partial x_{\alpha}, \partial x_{\beta}\right)$, and $R^{P}$ denotes the scalar curvature of $P$. In particular, if $\operatorname{dim} P=n-1$, or equivalently, if the isoparametric function $f$ is not proper, then (10) reduces to

$$
R^{N}-\rho^{N}(\nu, \nu)-R^{P}=\Gamma_{P}
$$

where $\nu$ is the unit normal vector of $P$. If in addition $N$ is an Einstein manifold, i.e., $\rho^{N} \equiv$ Const, then $P$ has constant scalar curvature. In conclusion, we get:

Corollary 3.1. On the unit normal bundle of the focal varieties $M_{ \pm}$of an isoparametric function $f$ on a complete Riemannian manifold $N$, the function $\Gamma_{P}$ defined in (9) is constant and the equality (10) holds. If in addition $N$ is Einstein and $f$ is isoparametric and not proper, then the "non-singular" focal variety is a minimal hypersurface with constant scalar curvature.

Proof of Theorem 1.2. As before, we can apply Corollary 2.2 in our case with $P=$ $M_{-}$(alternatively $M_{+}$). Now by assumption, $P_{t}$ has constant principal curvatures, say $\lambda_{1}(t), \cdots, \lambda_{n-1}(t)$, with respect to the unit normal vector field in the direction of $\nabla f$. All $\lambda_{i}(t)$ 's depend only on $t>0$, regardless of whether $P_{t}$ is connected or not. Indeed, when $P$ has codimension 1 , as a level hypersurface $P_{t}$ may still be disconnected. However, by Footnote $1.2, \lambda_{i}(t)$ 's depend only on $\left.f\right|_{P_{t}}$ which is a oneparameter function of $t$. Then by Corollary 2.2,

$$
\bar{S}(t)=\left(\begin{array}{cc}
T_{v} & 0 \\
0 & -\frac{1}{t} I
\end{array}\right)+t\left(\begin{array}{cc}
A & B \\
C & D
\end{array}\right)+\mathcal{O}\left(t^{2}\right)
$$

has $\lambda_{1}(t), \cdots, \lambda_{n-1}(t)$ as its eigenvalues. Let $\mu_{1}(v), \cdots, \mu_{m}(v)$ be the eigenvalues of $T_{v}$. Then without loss of generality, by (11) we can assume

$\lambda_{a}(t)=\mu_{a}(v)+O(t), \quad \lambda_{k}(t)=-\frac{1}{t}+O(t), \quad$ for $a=1, \cdots, m ; k=m+1, \cdots, n-1$.

Taking limit of $t$ to 0 , we have

$$
\mu_{a}(v)=\lim _{t \rightarrow 0+} \lambda_{a}(t)=: \lambda_{a} \text { (const) }
$$

for any $p \in P$ and unit normal vector $v \in \mathcal{V}_{p} P$, which completes the proof.

Note that for any unit normal vector $v \in \mathcal{V}_{p} P, T_{v}$ has the same constant eigenvalues $\lambda_{1}, \cdots, \lambda_{m}$, while by linearity, $T_{-v}=-T_{v}$. Thus the constants $\lambda_{a}$ must occur in pairs of opposite signs, i.e., $\kappa_{1},-\kappa_{1}, \cdots, \kappa_{q},-\kappa_{q}, 0, \cdots, 0$. Such submanifold with principal curvatures in any direction occurring in pairs of opposite signs is called austere submanifold in [HL82]. On the other hand, by the Riccati equation (6), we know that the Ricci curvature $\rho^{N}(\nu, \nu)$ is constant on each $P_{t}$ (where $\nu$ is the unit normal vector field) and thus

$$
\rho^{N}(v, v)=\lim _{t \rightarrow 0+} \rho^{N}(\nu, \nu)
$$

is constant on $P$ for any unit normal vector field $v$, which together with formulas (9, 12) imply:

$$
\sum_{a=1}^{m} K^{N}\left(v, \partial x_{a}\right) \equiv \text { const. }
$$


Thus if $N$ has constant scalar curvature on $P$, so does $P$ by formulas $(10,13)$ and the constancy of $\rho^{N}(v, v)$. In conclusion, we obtain:

Corollary 3.2. Suppose that each isoparametric hypersurface $M_{t}$ has constant principal curvatures. Then the Ricci curvature $\rho^{N}$ of $N$ is constant on each $M_{t}$ and the focal varieties $M_{ \pm}$in their normal directions. Moreover, the curvature identity (13) holds on the focal varieties. If in addition the scalar curvature of $N$ is constant on $M_{ \pm}$, then the focal varieties also have constant scalar curvature.

The curvature restrictions above could also be derived by analyzing the expansion formula (11) which may imply some subtler curvature relations (perhaps explicit higher order terms would be required).

Acknowledgements. It is our great pleasure to thank Professor Gudlaugur Thorbergsson for many useful discussions and kindly introductions of works on normal holonomy theory, and also thank Professor Yoshihiro Ohnita for his interests on our work. We would also like to thank the referees for their careful reviews and helpful comments.

\section{REFERENCES}

[BCO03] J. Berndt, S. Console, And C. Olmos, Submanifolds and holonomy, CRC/Chapman and Hall, Research Notes Series in Mathematics, 434, Boca Raton, 2003.

[Br99] M. BRÜCK, Equifocal families in symmetric spaces of compact type, J. Reine Angew. Math., 515 (1999), pp. 73-95.

[Car38] E. CARTAN, Familles de surfaces isoparamétriques dans les espaces à courbure constante, Annali di Mat., 17 (1938), pp. 177-191.

[Car39] E. CARTAN, Sur des familles remarquables d'hypersurfaces isoparamétriques dans les espaces sphériques, Math. Z., 45 (1939), pp. 335-367.

[CCJ07] T. E. CECIL, Q. S. CHI, AND G. R. JEnsen, Isoparametric hypersurfaces with four principal curvatures, Ann. Math., 166 (2007), pp. 1-76.

[CDO08] S. Console, A. J. Di Scala, and C. Olmos, A Berger type normal holonomy theorem for complex submanifolds, Math. Ann., 351 (2011), pp. 187-214.

[GT09] J. Q. GE AND Z. Z. TANG, Isoparametric functions and exotic spheres, J. Reine Angew. Math., 683 (2013), p. 161-180.

[GX10] J. Q. GE AND Y. Q. XIE, Gradient map of isoparametric polynomial and its application to Ginzburg-Landau system, J. Funct. Anal., 258 (2010), pp. 1682-1691.

[Gr04] A. Gray, Tubes, Second Edition, Progress in Mathematics, Vol.221. Birkhäuser Verlag Basel, Boston, Berlin (2004).

[HL82] R. HARvey AND B. LAwson, Calibrated geometries, Acta Math., 148 (1982), pp. $47-157$.

[Imm08] S. IMMERVOLL, On the classification of isoparametric hypersurfaces with four distinct principal curvatures in spheres, Ann. Math., 168 (2008), pp. 1011-1024.

[Mmp06] F. Mahmoudi, R. Mazzeo And F. PACARd, Constant mean curvature hypersurfaces condensing on a submanifold, Geom. Funct. Anal., 16 (2006), pp. 924-958.

[MP05] R. MAzzeo AND F. PACARD, Foliations by constant mean curvature tubes, Comm. Anal. Geom., 13 (2005), pp. 633-670.

[Mi12] R. MiyaokA, Transnormal functions on a Riemannian manifold, Diff. Geom. Appl., 31 (2013), pp. 130-139.

[Mü80] H. F. MünZner, Isoparametric hyperflächen in sphären, I and II, Math. Ann., 251 (1980), pp. 57-71 and 256 (1981), pp. 215-232.

[Ni97] L. NI, Notes on Transnormal Functions on Riemannian Manifolds, unpublished, 1997, available at: http://math.ucsd.edu/ lni/academic/isopara.pdf.

[No73] K. Nomizu, Some results in E. Cartan's theory of isoparametric families of hypersurfaces, Bull. Amer. Math. Soc., 79 (1973), pp. 1184-1189.

[Th00] G. Thorbergsson, A survey on isoparametric hypersurfaces and their generalizations, in Handbook of differential geometry, Vol. I, North - Holland, Amsterdam, (2000), pp. 963-995.

[Wa87] Q. M. WANG, Isoparametric Functions on Riemannian Manifolds. I, Math. Ann., 277 (1987), pp. 639-646. 
J. Q. GE AND Z. Z. TANG 